\title{
Floral Bud and Mean Petal Length as Morphological Predictors of Microspore Cytological Stage in Lesquerella
}

\author{
Pernell Tomasi ${ }^{1}$ and David A. Dierig ${ }^{2}$ \\ U.S. Department of Agriculture, Agricultural Research Service, U.S. Water \\ Conservation Laboratory, 4331 East Broadway Road, Phoenix, AZ 85040-8832
}

\author{
Ralph A. Backhaus ${ }^{3}$ and Kathleen B. Pigg ${ }^{4}$ \\ Department of Plant Biology, P.O. Box 871601, Arizona State University, \\ Tempe, AZ 85287-1601
}

Additional index words. androgenesis, Lesquerella fendleri, microsporogenesis, morphometrics

Obtaining homozygous lines from highly outcrossing, self-incompatible species requires multiple generations of time-consuming bud pollinations. The utilization of microspore embryogenesis by anther or pollen culture can generate purely homozygous lines more rapidly and efficiently than can conventional means, thus allowing for mutational breeding and selection of beneficial crop traits. However, in many instances, androgenic cultures of novel species exhibit limited embryo yields or fail to develop altogether. One of the most crucial parameters for success is obtaining the proper stage of microspore development at culture initiation (Chuong et al., 1988a).

Lesquerella [Lesquerella fendleri (Gray) Wats.], a member of the Brassicaceae, is a diploid $(n=6)$ species that exhibits sporophytic self-incompatibility with an outcross-

Received for publication 1 Feb. 1999. Accepted for publication 14 June 1999. This paper represents a thesis presented in partial fulfillment of the requirements for the degree Master of Science in Plant Biology. The cost of publishing this paper was defrayed in part by the payment of page charges. Under postal regulations, this paper therefore must be hereby marked advertisement solely to indicate this fact.

${ }^{1}$ Research Technician. E-mail address: ptomasi@ uswcl.ars.ag.gov

${ }^{2}$ Research Geneticist.

${ }^{3}$ Professor.

${ }^{4}$ Associate Professor. ing rate of $86 \%$ to $89 \%$ (Dierig et al., 1996). This creates difficulties when trying to produce homozygous breeding lines necessary for improvement of yield-related traits. Lesquerella has recently been introduced from the wild for development as a source of hydroxy fatty acids, specifically used for industrial products such as coatings, lubricants, and greases. To our knowledge, no effective microspore culture protocol has been developed for this potentially useful, new industrial oilseed crop. The objective of this study was to correlate the stages of microspore development in lesquerella with other morphometric attributes of the plant in order to better predict the proper timing of culture initiation.

\section{Materials and Methods}

This study was performed during the 199697 and 1997-98 crop season at the U.S. Dept. of Agriculture (USDA)-Agricultural Research Service(ARS), U.S. Water Conservation Laboratory, Phoenix, Ariz. Lesquerella plants were derived from a synthetic population of nine accessions (PI 293005, PI 293006, PI 293007, PI 293009, PI 293010, PI 293012, PI 293013, PI 293015, PI 293016, and PI 331165) described by Thompson and Dierig (1994). A total of 170 greenhouse-grown floral buds were harvested in 1997 and 1998 from lesquerella breeding lines L97-14, L97-22, L97-47, and L98-53. The lengths of each individual floral bud, four petals (two large and two small), and six anthers (four large and two small) were measured with a micrometer caliper.

Anthers from each bud were bulked, squashed, and fixed with $0.05 \%$ toluidine blue in McIlvaine buffer solution $(\mathrm{pH} \mathrm{7.0)}$ and observed microscopically (Chuong et al., 1988b). Each individual anther squash was assigned an arbitrary value according to the nuclear stage present as described by Kott et al. (1988), viz., $1=$ tetrad, $2=$ early uninucleate, $3=$ mid-uninucleate, $4=$ late uninucleate, and $5=$ vegetative and generative (Fig. 1). A minimum of 100 observations were made for each preparation. Cytological data from the four lines were used to determine pooled and by-line Pearson's correlation coefficients (SAS Institute, 1995) between each of the floral morphometric variables and corresponding microspore stage values. Each floral parameter was averaged and the means separated by the Student-Newman-Keuls' mean separation statistic (SAS Institute, 1995) to establish pooled categorical stage classifications. Linear regression values were calculated to determine microspore developmental stage prediction equations based on floral bud length measurements.

\section{Results and Discussion}

All measured morphometric parameters were significantly correlated with microspore stage $(P \leq 0.0001)$ for the pooled analysis $(\mathrm{n}=$ $170)$ with the most significant correlation being the mean length of the two largest petals $(r$ $=0.80$ ). Further by-line analysis revealed that the bud size of line L97-14 was highly correlated $(r=0.84)$ with microspore stage, whereas in lines L97-22 and 47 and L98-53, the mean length of the two largest petals was a better predictor of microspore stage $(r=0.72,0.92$, and 0.81 , respectively). Distinct classes of microspore developmental stages were apparent for both floral bud and petal length (Table 1). Other androgenic protocols for Brassica suggest that the late uninucleate stage of microspore development (stage 4) is the most responsive in culture (Coventry et al., 1988; Keller, 1984). If lesquerella is considered similarly, the selection of pooled floral buds 3.7 to $4.0 \mathrm{~mm}$ in length, with mean petal lengths of 2.7 to $3.4 \mathrm{~mm}$, would assure that isolated microspores were at the late uninucleate stage

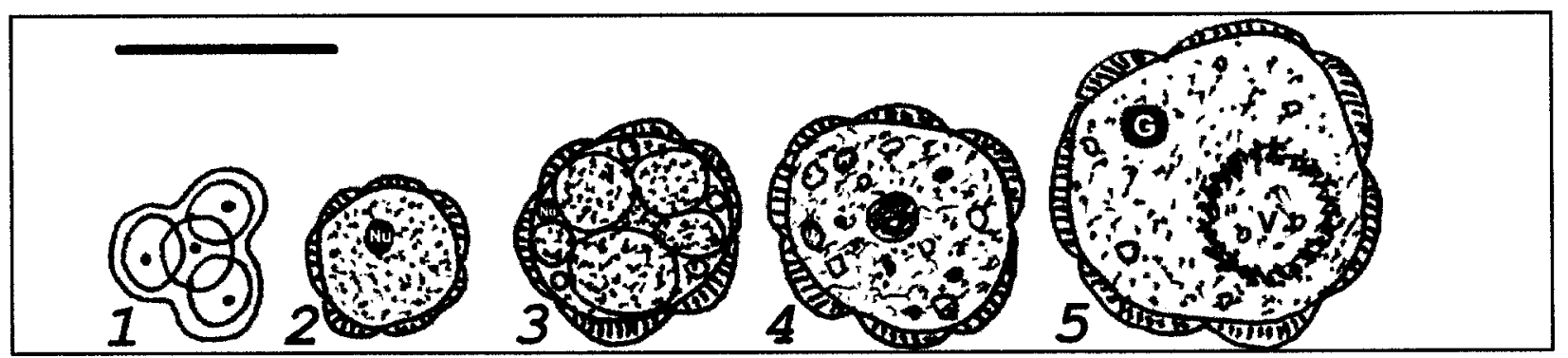

Fig. 1. Cytological stages of L. fendleri microspores: 1) tetrad structure of meiotic division; 2) uninucleate microspore with prominent nucleolus (Nu) and exine lobes; 3) mid-uninucleate microspore vacuolation; 4) late uninucleate microspore with multiple vacuoles and diffuse nucleolus; 5) binucleate pollen grain with distinct generative $(\mathbf{G})$ and vegetative $(\mathbf{V})$ nuclei. $\mathrm{Bar}=20 \mathrm{um}$. 
Table 1. Establishment of categorical stage rank classifications based upon morphological measurements of lengths of floral bud and petals of 170 pooled, greenhouse-grown lesquerella lines in 1997 and 1998 .

\begin{tabular}{lcccc}
\hline & & & \multicolumn{2}{c}{ Length $(\mathrm{mm})^{\mathrm{z}}$} \\
\cline { 3 - 4 } Stage & Nuclear stage of microspore & $\mathrm{N}$ & Floral bud & Petals \\
\hline 2.0 & Early uninucleate & 43 & $3.13 \pm 0.40 \mathrm{a}^{\mathrm{y}}$ & $1.69 \pm 0.45 \mathrm{a}$ \\
3.0 & Mid-uninucleate & 62 & $3.48 \pm 0.37 \mathrm{~b}$ & $2.31 \pm 0.43 \mathrm{~b}$ \\
4.0 & Late uninucleate & 34 & $3.70 \pm 0.43 \mathrm{c}$ & $2.74 \pm 0.50 \mathrm{c}$ \\
5.0 & Vegetative/generative & 31 & $4.05 \pm 0.40 \mathrm{~d}$ & $3.42 \pm 0.44 \mathrm{~d}$
\end{tabular}

${ }^{\mathrm{z}}$ Means $\pm \mathrm{sE}$

${ }^{y}$ Mean separation within columns by Student-Newman-Keuls' test at $P \leq 0.05$.

of development. Concurrent lesquerella anther cultures and microspore isolations utilized floral bud sizes ranging from 1.77 to 5.59 $\mathrm{mm}$. This range includes all the microspore developmental stages, but, unfortunately, no microspore-derived embryos were initiated following either of the two attempted culture protocols.

Floral bud phenotypes were more variable for line L97-22 than for the other three lines. The floral buds of this line had irregular sepal shapes and lengths. Floral buds from line L97-22 were collected in late April, near the end of the 1996-97 lesquerella season when greenhouse temperatures were higher, possibly resulting in more variable floral bud morphology and a heterogeneous mixture of microspore stages (Reinhert and Bajaj, 1977). Statistical analysis supported visual observations of greater phenotype dissimilarity in L97-22 $\left(r^{2}=0.19\right)$ than in lines L97-14 $\left(r^{2}=\right.$ $0.70)$, L97-47 $\left(r^{2}=0.63\right)$, or L98-53 (hybrid between lines L97-14 and 47, $r^{2}=0.58$ ).

Our results show that morphological parameters of floral bud and mean petal lengths can reliably be used to predict microspore developmental stage in pooled samples and some individual lines of lesquerella. These analyses demonstrate that the labor-intensive, aseptic measurement of all six anther and four petal lengths is unnecessary for determining microspore cytological stages in lesquerella. A reliable estimation of microspore developmental stage can be attained by measuring the lengths of the floral bud and the two largest petals, with the other morphological measurements being inconsequential. The measurement of only these two floral attributes will allow for more rapid bud staging in future microspore culture trials. To maintain isolation efficiency and reduce the chance of contamination, the floral bud length should be used to adequately predict microspore cytological stage.

\section{Literature Cited}

Chuong, P.V., C. Deslauriers, L.S. Kott, and W.D. Beversdorf. 1988a. Effects of donor genotype and bud sampling on microspore culture of Brassica napus. Can J. Bot. 66:1653-1657.

Chuong, P.V., K.P. Pauls, and W.D. Beversdorf, 1988b. High-frequency embryogenesis in male sterile plants of Brassica napus through microspore culture. Can J. Bot. 66:1676-1680.

Coventry, J., L. Kott, and W.D. Beversdorf. 1988 Manual for microspore culture technique for Brassica napus. Univ. Of Guelph, Ont., Dept. of Crop Sci. Bul. 0489.

Dierig, D.A., T.A. Coffelt, F.S. Nakayama, and A.E Thompson. 1996. Lesquerella and vernonia: Oilseeds for arid lands, p. 347-354. In: J. Janick (ed.). Progress in new crops. ASHS Press, Alexandria, Va.

Keller, W.A. 1984. Anther culture of Brassica, p. 302-310. In: I.K. Vasil (ed.). Cell culture and somatic cell genetics of plants. Vol.1. Academic Press, New York.

Kott, L.S., L. Polsoni, and W.D. Beversdorf. 1988. Cytological aspects of isolated microspore culture of Brassica napus. Can. J. Bot. 66:16581664.

Reinert J. and Y.P.S. Bajaj, 1977. Anther culture: haploid production and its significance, p. 251267. In: J. Reinert and Y. P. S. Bajaj (eds.). Applied and fundamental aspects of plant cell, tissue, and organ culture. Narosa Publ. House, New Delhi.

SAS Institute. 1995. SAS user's guide: Statistics. ver. 6.11. SAS Inst., Cary, N.C.

Thompson, A.E. and D.A. Dierig. 1994. Initial selection and breeding of Lesquerella fendleri, a new industrial oilseed. Ind. Crops Prod. 2:97-106. 